\title{
Paralisia periódica hipocalêmica tireotóxica em paciente de ascendência africana
}

\author{
Thyrotoxic hypokalemic periodic paralysis in patients of African descent
}

Morgana Lima e Maia', Paula Grasiele Carvalho Trevisam', Marcos Minicucci', Glaucia M. F. S. Mazeto', Paula S. Azevedo'

\section{SUMÁRIO}

A paralisia periódica hipocalêmica tireotóxica (PPHT) é uma urgência endocrinológica caracterizada por ataques recorrentes de fraqueza muscular, associados à hipocalemia e à tireotoxicose. Essa patologia ocorre mais frequentemente em pacientes do sexo masculino, de origem asiática, sendo rara sua apresentação em pessoas de ascendência africana. 0 caso descrito mostra um paciente afrodescendente que apresentou tetraparesia associada à hipocalemia. Durante esse episódio foi realizado o diagnóstico de hipertireoidismo. A PPHT, embora rara, é potencialmente letal. Portanto, em casos de crises de paresias flácidas, essa hipótese diagnóstica deve ser sempre considerada, principalmente se associada à hipocalemia. Nessa situação, se não houver diagnóstico prévio de hipertireoidismo, este também deve ser considerado. Arq Bras Endocrinol Metab. 2014;58(7):779-81

\section{SUMMARY}

Thyrotoxic hypokalemic periodic paralysis (THPP) is an endocrine emergency marked by recurrent attacks of muscle weakness associated with hypokalemia and thyrotoxicosis. Asiatic male patients are most often affected. On the other hand, African descents rarely present this disease. The case described shows an afrodescendant patient with hypokalemia and tetraparesis, whose diagnosis of hyperthyroidism was considered during this crisis. The THPP, although rare, is potentially lethal. Therefore, in cases of flaccid paresis crisis this diagnosis should always be considered, especially if associated with hypokalemia. In this situation, if no previous diagnosis of hyperthyroidism, this should also be regarded. Arq Bras Endocrinol Metab. 2014;58(7):779-81
'Departamento de Clínica Médica, Faculdade de Medicina de Botucatu, Universidade Estadual Paulista Júlio de Mesquita Filho (Unesp), Botucatu, SP, Brasil

\section{INTRODUÇÃO}

$\mathrm{P}$ aralisia periódica hipocalêmica tireotóxica (PPHT) é uma complicação potencialmente letal da tireotoxicose, caracterizada por ataques agudos de fraqueza muscular associados a diferentes níveis de hipocalemia $(1,2)$. Comumente acomete homens entre 20 e 40 anos de idade e de ascendência asiática. Entretanto, pode ser encontrada em outras etnias, com menor frequência (2).

A fisiopatologia da PPHT é multifatorial composta por suscetibilidade genética, presença de tireotoxicose e fatores ambientais (2).

Frequentemente, a PPHT é subdiagnosticada por características de sua apresentação clínica. Adicionalmente, há diversos diagnósticos diferenciais para crises de fraqueza muscular, tais como paralisia periódica hipocalêmica familiar, síndrome de Guillain-Barré, distúrbios de eletrólitos e doenças neurológicas e musculares (1-3).

O tratamento na sala de emergência consiste na correção cuidadosa da hipocalemia e controle dos sintomas e sinais da tireotoxicose $(4,5)$. Posteriormente, o tratamento definitivo do hipertireoidismo é fundamental para prevenir novas crises $(2,4)$.

Apresentamos o caso de um paciente afrodescendente, sem diagnóstico prévio de hipertireodismo, que apresentou episódio agudo de PPHT, com regressão completa do quadro, após reposição de potássio e controle da tireotoxicose. 


\section{RELATO DO CASO}

Paciente de 37 anos, gênero masculino, de ascendência negra, apresentou quadro clínico de adinamia, hiporexia, tremor, diarreia e perda ponderal de 10 $\mathrm{kg}$, que se iniciou 30 dias antes de procurar o serviço médico. Adicionalmente, apresentou fraqueza em membros inferiores (MMII), com sensação de peso e dificuldade de deambulação por 10 dias. Procurou setor de emergência 3 horas após iniciar paresia dos membros superiores (MMSS) e piora dos sintomas dos membros inferiores. $\mathrm{O}$ exame físico geral mostrou frequência cardíaca de 96 bpm; pressão arterial de 200 x $60 \mathrm{mmHg}$, frequência respiratória de $20 \mathrm{mpm}$; saturação periférica de oxigênio de $98 \%$, em ar ambiente. O exame neurológico evidenciou discreta hipotonia de MMII; arreflexia patelar e de aquileu à esquerda e hiporreflexia à direita; força muscular (escala Medical Research Council) grau 3 proximal e grau 1 distal em MMII e grau 5 em MMSS. Após 2 horas, houve piora da paresia de MMII e em MMSS, grau 1 bilateralmente, tornando-se tetraparético. $\mathrm{O}$ exame físico dos demais sistemas não mostrou alterações significativas.

Os exames laboratoriais de urgência mostraram potássio 1,9 mEq/L (VR: 3,5-5,1), sódio $143 \mathrm{mEq} / \mathrm{L}$ (VR: 137-145 mEq/L), ureia $39 \mathrm{mg} / \mathrm{dL}$ (VR: 15-37 $\mathrm{mEq} / \mathrm{L}$ ), creatinina $0,8 \mathrm{mg} / \mathrm{dL}$ (VR: $0,7-1,2 \mathrm{mE}-$ $\mathrm{q} / \mathrm{L})$. A reposição de potássio endovenoso foi realizada e houve melhora do quadro neuromuscular e normalização do potássio sérico $(4,6 \mathrm{mEq} / \mathrm{L})$.

Foram solicitados outros exames laboratoriais na rotina: TSH $0,00 \mathrm{mUI} / \mathrm{mL}(0,4-4,0)$; T4 livre 4,85 ng/dL (0,8-1,9); antitireoglobulina 5,11 UI/mL (014,4); antiTPO 92,42 UI/mL (0-5,6); TRAB 17,96 UI/L (até 1,75). A ultrassonografia de tireoide mostrou discreto aumento da glândula, com sinais de tireopatia parenquimatosa difusa, com fluxo vascular aumentado. A cintilografia de tireoide com pertecnetato de tecnécio evidenciou bócio difuso hipercaptante.

Assim, o diagnóstico de hipertireoidismo por doença de Graves associado à PPHT foi confirmado. O tratamento medicamentoso da tireotoxicose com propranolol e metimazol foi iniciado, com desaparecimento de todos os demais sintomas. Atualmente, o paciente encontra-se em uso de $15 \mathrm{mg} /$ dia de metimazol e 40 mg 12/12 horas de propranolol e aguarda tratamento definitivo do hipertireoidismo.

\section{DISCUSSÃO}

A PPHT é uma complicação potencialmente letal do hipertireoidismo, caracterizada por ataques agudos de fraqueza muscular, tireotoxicose e hipocalemia $(1,2)$.

Considerando os dados epidemiológicos, o quadro afeta principalmente pacientes do sexo masculino, de origem asiática, entre 20 e 40 anos de idade $(2,6)$. Porém, nas últimas décadas, um número crescente de pacientes de todas as etnias tem sido relatado (2). Em casuística brasileira, Maciel e cols. observaram que, em 33 pacientes com PPHT, dois eram de ascendência afro-americana e 11 pacientes apresentavam ascendência mista de brancos e negros (2). O caso descrito refere-se a paciente de ascendência negra, ressaltando a importância de se pensar em PPHT, independentemente da raça ou região geográfica.

A PPHT é subdiagnosticada, pois muitas vezes os pacientes apresentam quadro clínico de tireotoxicose, mas não possuem diagnóstico prévio $(2,7)$. Desse modo, é comum que o diagnóstico de hipertireoidismo seja realizado concomitantemente à PPHT. Adicionalmente, existem diversos diagnósticos diferenciais para o quadro de fraqueza muscular, que variam de doenças musculares ou neurológicas até transtornos de ansiedade. De fato, em estudo prévio observou-se que alguns pacientes que apresentavam fraqueza muscular foram tratados inicialmente com benzodiazepínicos (2).

Em relação ao quadro clínico da PPHT, este é caracterizado por episódios recorrentes e transitórios de fraqueza muscular, que variam de leve a acentuados. A apresentação clínica pode ser caracterizada por paresia ou paralisia flácida, sendo os músculos proximais mais afetados que os distais (8). Em geral, as alterações musculares acometem primeiramente os MMII, com progressão ascendente, a função sensitiva não é afetada e os músculos podem ser acometidos de forma assimétrica $(8,9)$. Na maioria dos pacientes, os reflexos tendinosos profundos são marcadamente diminuídos ou ausentes. Alguns casos, no entanto, apresentam reflexos normais (9). A PPHT manifesta-se mais comumente no período noturno ou pela manhã, principalmente após ingestão de grandes quantidades de carboidratos ou durante o repouso após atividade física, que favorecem a secreção de insulina (2). Esse quadro é associado a hipocalemia, que pode ou não ser detectada no momento da crise, $\mathrm{e}$ sintomas ou sinais de tireotoxicose, ainda que discretos.

No caso descrito anteriormente, o paciente apresentou quadro de fraqueza muscular ascendente, culminando com tetraparesia flácida, associado a hipocalemia, diarreia e perda de peso. Em decorrência de presença 
de tetraparesia flácida, uma das hipóteses aventadas foi a síndrome de Guillain-Barré. Outro raciocínio clínico aplicado ao caso foi que a diarreia e um possível quadro de desidratação poderiam justificar a hipocalemia. Além disso, diarreia e perda de peso, embora inespecíficos, podem fazer parte do quadro clínico de tireotoxicose. Portanto, tetraparesia flácida, hipocalemia e possível tireotoxicose permitiram a hipótese diagnóstica de PPHT. Em seguida, com diagnóstico de hipertireoidismo confirmado com exames complementares, confirmou-se também o diagnóstico de PPHT.

Sobre a fisiopatologia da PPHT, é possível que o excesso de hormônios tireoidianos, a estimulação $\beta 2$ adrenérgica e a hiperinsulinemia estimulem a bomba de sódio/potássio ATPase, localizada nas membranas celulares dos músculos esqueléticos, resultando em transporte de potássio para o compartimento intracelular, com consequente hipocalemia e alterações na força muscular $(7,10)$. Entretanto, a patogênese da PPHT não é totalmente elucidada, sendo possível que haja também predisposição genética (1). Nos últimos anos, estudos foram realizados com o intuito de identificar mutações ou polimorfismos genéticos que predispõem a PPHT. Em 2010, Ryan e cols. descreveram que mutações no canal de potássio Kir2.6 podem predispor a PPHT (11). Essas mutações favorecem disfunção dos canais de potássio, alterando o potencial de ação e a contração muscular, com consequente paralisia flácida (2).

Por fim, o tratamento deve ser realizado com a suplementação imediata de potássio que previne complicações cardiorrespiratórias graves e pode acelerar a recuperação de fraqueza muscular (4). Faz-se importante destacar que a hipocalemia acontece por redistribuição do potássio do compartimento extracelular para o intracelular. Dessa forma, os estoques de potássio permanecem íntegros e sua concentração sérica deve se normalizar após o controle da crise. Assim, a reposição de potássio deve ser cuidadosa e preferencialmente pela via oral, para evitar hipercalemia de rebote após a resolução da crise. Adicionalmente, betabloqueadores não seletivos podem aliviar e prevenir a recorrência dos episódios de paralisia $(4,5)$. De fato, os betabloqueadores como o propranolol têm sido apontados como drogas de escolha para o tratamento da PPHT por bloquear a bomba de sódio/potássio ATPase. Consequentemente, essas drogas auxiliam na recuperação da força muscular e na correção da hipocalemia, sem que haja hipercalemia de rebote. No caso descrito, o paciente recebeu potássio pela via endovenosa, pois concentrações desse micronutriente abaixo de $2,0 \mathrm{mEq} / \mathrm{L}$ são consideradas como hipocalemia grave, sendo necessária maior urgência na reversão do quadro.
Além dessas medidas, faz-se necessário o controle do hipertireoidismo, com uso de medicamentos antitireoidianos, iodo radioativo ou cirurgia (2). É importante lembrar que a crise acontece quando o paciente está em tireotoxicose. Dessa forma, o tratamento definitivo para o hipertireoidismo deve ser recomendado. No caso em questão, após a confirmação diagnóstica do hipertireoidismo, foi mantido o betabloqueador e iniciado metimazol, com controle adequado da tireotoxicose e programação para tratamento definitivo desta.

Em conclusão, a PPHT é uma condição rara, porém potencialmente letal. Embora seja mais prevalente em pacientes de ascendência asiática, pode acometer pacientes com tireotoxicose de qualquer etnia. A PPHT muitas vezes é subdiagnosticada ou é diagnosticada tardiamente, por fatores como quadro clínico frustro de tireotoxicose, potássio sérico normal, outros diagnósticos diferenciais, entre outros.

Desse modo, em casos de ataques agudos de fraqueza muscular, deve-se aventar a hipótese de PPHT, independentemente da etnia do paciente, mesmo na ausência de hipocalemia e do diagnóstico prévio de hipertireoidismo. O controle adequado do hipertireoidismo deve ser mandatório e seu tratamento definitivo deve ser recomendado para prevenção de novas crises.

\section{REFERÊNCIAS}

1. Falhammar $\mathrm{H}$, Thoren $\mathrm{M}$, Calissendorff $\mathrm{J}$. Thyrotoxic periodic paralysis: clinical and molecular aspects. Endocrine. 2013;43:274-84.

2. Maciel RM, Lindsey SC, Dias da Silva MR. Novel etiopathophysiological aspects of thyrotoxic periodic paralysis. Nat Rev Endocrinol. 2011;7:657-67.

3. Barnabé C. Acute generalized weakness due to thyrotoxic periodic paralysis. CMAJ. 2005;172:471-2.

4. Tassone $H$, Moulin A, Henderson SO. The pitfalls of potassium replacement in thyrotoxic periodic paralysis: a case report and review of the literature. J Emerg Med. 2004;26:157-61.

5. CopeTE, Samaraweera AP, Burn DJ.Thyrotoxic periodic paralysis: correct hypokalemia with caution. J Emerg Med. 2013;45:338-40.

6. Silva MR, Chiamolera MI, Kasamatsu TS, Cerutti JM, Maciel RM. [Thyrotoxic hypokalemic periodic paralysis, an endocrine emergency: clinical and genetic features in 25 patients]. Arq Bras Endocrinol Metabol. 2004;48:196-215.

7. Pothiwala $P$, Levine SN. Analytic review: thyrotoxic periodic paralysis: a review. J Intensive Care Med. 2010;25:71-7.

8. Kung AW. Clinical review: thyrotoxic periodic paralysis: a diagnostic challenge. J Clin Endocrinol Metab. 2006;91:2490-5.

9. Kelley DE, Gharib H, Kennedy FP, Duda RJ Jr, McManis PG. Thyrotoxic periodic paralysis. Report of 10 cases and review of electromyographic findings. Arch Intern Med. 1989;149:2597-600.

10. Lin $\mathrm{SH}$, Huang $\mathrm{CL}$. Mechanism of thyrotoxic periodic paralysis. J Am Soc Nephrol. 2012;23:985-8.

11. Ryan DP, da Silva MR, Soong TW, Fontaine B, Donaldson MR, Kung AW, et al. Mutations in potassium channel Kir2.6 cause susceptibility to thyrotoxic hypokalemic periodic paralysis. Cell. 2010;140:88-98. 